\title{
BMJ Open How much of the effect of disability acquisition on mental health is mediated through employment and income? A causal mediation analysis quantifying interventional indirect effects using data from four waves of an Australian cohort study
}

\author{
Zoe Aitken (D) ,' Julie Anne Simpson, ${ }^{2}$ Rebecca Bentley (D) , ${ }^{3}$ \\ Anne Marie Kavanagh (i) ${ }^{1}$
}

To cite: Aitken Z, Simpson JA, Bentley R, et al. How much of the effect of disability acquisition on mental health is mediated through employment and income? A causal mediation analysis quantifying interventional indirect effects using data from four waves of an Australian cohort study. BMJ Open 2021;11:e055176. doi:10.1136/ bmjopen-2021-055176

- Prepublication history and additional supplemental material for this paper are available online. To view these files, please visit the journal online (http://dx.doi.org/10.1136/ bmjopen-2021-055176).

Received 05 July 2021 Accepted 02 November 2021

Check for updates

(C) Author(s) (or their employer(s)) 2021. Re-use permitted under CC BY-NC. No commercial re-use. See rights and permissions. Published by BMJ.

For numbered affiliations see end of article.

Correspondence to

Dr Zoe Aitken;

zoe.aitken@unimelb.edu.au

\section{ABSTRACT}

Objectives There is evidence that disability acquisition causes a decline in mental health, but few studies have examined the causal mechanisms through which the effect operates. This study used a novel approach to mediation analysis to quantify interventional indirect effects (IIEs) through employment and income.

Design and setting We used four waves of longitudinal data (2011-2014) from the Household, Income and Labour Dynamics in Australia Survey, a nationally representative survey of Australian households.

Participants Working aged individuals who acquired a disability $(n=233)$ were compared with those who remained disability-free in all four waves $(n=5419)$.

Primary outcome measure Self-reported mental health was measured using the Mental Health Inventory subscale of the Short Form 36 general health questionnaire, which measures symptoms of depression, anxiety and psychological well-being.

Statistical analysis We conducted a causal mediation analysis quantifying IIEs of disability acquisition on mental health operating through two distinct mediators: employment status and income. We used multiple imputation with 50 imputed datasets to account for missing data.

Results The total causal effect of disability acquisition on mental health was estimated to be a 4.8-point decline in mental health score (estimated mean difference: -4.8 , $95 \% \mathrm{Cl}-7.0$ to -2.7$)$. The IIE through employment was estimated to be a 0.5 -point difference $(-0.5,95 \% \mathrm{Cl}-1.0$ to 0.0 ), accounting for $10.6 \%$ of the total effect, whereas there was no evidence that income explained any of the effects.

Conclusions This study estimated that disabilityrelated mental health inequalities could be reduced by $10.6 \%$ if employment rates were the same for people with disability as those without disability. The results suggest that employment is implicated in the relationship between disability acquisition and mental health and that
Strengths and limitations of this study

This study uses data from a large nationally representative cohort study to examine how much of the effect of disability acquisition on mental health is mediated through employment and income.

- The longitudinal nature of the data was used to establish a temporal sequence between the baseline variables, exposure, mediators and outcomes to better model causal relationships.

- Interventional effects quantify average populationlevel effects representing the effect of shifting the distribution of the mediator in the population to what would be expected if everyone in the population was exposed-a more realistic estimate than natural effects which estimate individual causal effects.

- Causal mediation analysis relies on strong assumptions about confounding for the estimates to have a causal interpretation; however, there may have been some residual mediator-outcome confounding.

- Other limitations include: only 233 people acquired a disability resulting in low power to detect effects; variables were self-reported and therefore prone to measurement error; people with severe disabilities are likely to be under-represented in the sample.

more research is needed to understand the influence of other aspects of employment and other socioeconomic characteristics.

\section{INTRODUCTION}

In Australia, $18 \%$ of the population lives with a disability. ${ }^{1}$ People with disabilities experience large health inequalities compared with those without disability, a large proportion of which are unrelated to the impairment causing the 
disability. ${ }^{2-5}$ They report poorer mental health, including high rates of depression and anxiety, psychological distress and lower mental well-being. ${ }^{6-13}$ The relationship between disability and poor mental health is likely to be bidirectional. ${ }^{14}$ Several longitudinal studies have quantified the causal effect of disability on mental health, using repeated measures data and examining incident disability to isolate the direction of effect. ${ }^{71-13} 15-18$ These studies have provided evidence that disability leads to a deterioration in people's mental health, but the underlying mechanism is poorly understood. ${ }^{1920}$ This is an important omission because understanding the mechanism may highlight targets for interventions to mitigate the adverse effects of disability on mental health. ${ }^{21}$

There are a number of different mechanisms which could explain disability-related mental health inequalities. One hypothesis, supported by the social model of disability, is that health inequalities exist as a result of the environment and circumstances in which people with disabilities live. Therefore, inequalities are likely to operate through socioeconomic pathways.

In a previous analysis using the same data, we quantified how much of the effect of disability acquisition on mental health operated through three broad socioeconomic pathways and found that $32 \%$ of the effect was explained by material factors, with a negligible proportion of the effect explained by psychosocial or behavioural factors. ${ }^{20}$ Seven different material socioeconomic characteristics were examined jointly including employment, occupation, income, financial hardship, financial satisfaction, housing tenure and housing affordability. There remains a lack of understanding about which specific material factors drive the effect, limiting the development of effective interventions to improve the mental health of people with disabilities.

Novel causal mediation methods, known as interventional effects, allow quantification of indirect effects through distinct pathways in the presence of multiple mediators and can model the effects of population-level interventions. ${ }^{22}{ }^{23}$ In this study, we aim to further disentangle the mechanism linking disability and poor mental health, quantifying interventional effects through individual socioeconomic factors. We chose to examine two characteristics amenable to policy intervention: employment and income.

\section{METHODS}

\section{Data source}

We used data from the Household, Income and Labour Dynamics in Australia (HILDA) Survey, a large longitudinal study of Australian households, which collects information on a wide range of demographic, social, economic and health characteristics. ${ }^{22}$ The initial survey was conducted in 2001 with annual follow-up surveys. The original sample included 13969 participants from 7682 households, randomly sampled using a national probability sample of private dwellings. A top-up sample was

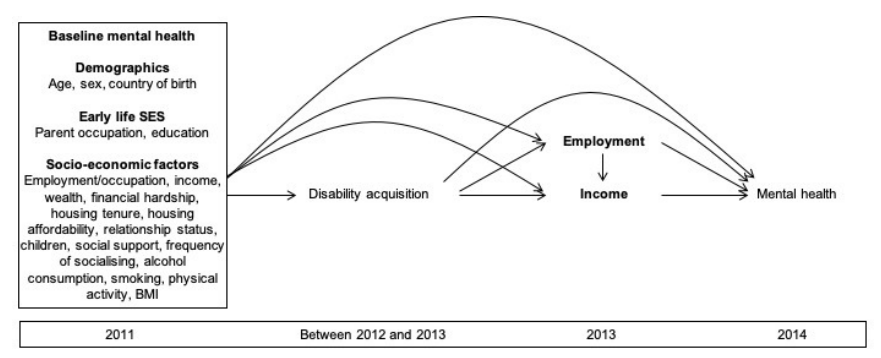

Figure 1 Causal diagram illustrating postulated causal relationships between disability acquisition and mental health. BMI, body mass index; SES, socioeconomic status.

added in 2011 to maintain representativeness. On average across all waves of the survey, response rates were $80 \%$ and attrition was $6 \%$ between waves. At each wave of the HILDA Survey, information about the survey is provided to new and existing study members in a brochure. By agreeing to be interviewed for the HILDA Survey, respondents provided implied consent to participate in the Survey. For children aged 15-17 years, verbal consent is provided by their parent or guardian.

This analysis was restricted to working age participants (25-64 years) because of the interest in examining pathways through employment. Data across four waves (2011-2014) were used to establish a temporal sequence between baseline characteristics (2011), disability acquisition (occurring between 2012 and 2013), the mediators (2013) and mental health (2014). The choice of variables included in the analyses was informed by the causal diagram (figure 1).

\section{Mental health}

Mental health was assessed in 2014 using the Mental Health Inventory (MHI), a subscale of the Short Form 36 (SF-36) health questionnaire. The MHI is a well-validated and reliable measure of mental health. ${ }^{24}$ It measures symptoms of depression, anxiety and psychological wellbeing and has been shown to be psychometrically sound ${ }^{25}$ and an effective screening tool for mood and anxiety disorders and severe depressive symptomatology. ${ }^{26-29}$ The MHI includes five items relating to mental health over the previous 4 weeks, each scored using five response categories. Total scores are transformed into a scale with a mean score of 74 (range: $0-100$ ), with higher scores reflecting better mental health. Previous research has suggested a difference of four to five points reflects a minimally important clinical difference in mental health. ${ }^{30} 31$

\section{Disability acquisition}

Information on disability was collected at every wave using a single question defining disability as 'an impairment, disability or long-term health condition, which restricts everyday activities that had lasted for six months or more'. Disability acquisition was defined as two waves reporting no disability (2011 and 2012), followed immediately by two consecutive waves reporting a disability (2013 and 2014). Two consecutive waves of disability were used to exclude people with transient disability, a definition 
used in previous studies. ${ }^{32-34}$ Participants who acquired a disability were compared with those who reported no disability in any of the four waves. Any other patterns of exposure, such as a single wave of disability, were excluded. Eligibility for inclusion required participation and response to the disability question at all four waves.

\section{Mediators}

The mediator variables, employment status and income were measured in 2013. Employment was categorised as a binary variable, defined as currently employed vs not employed (unemployed (actively seeking employment) or not in the labour force). Income was measured as a continuous variable describing people's weekly disposable income (in $\$ \mathrm{~A}$ ) truncated at the 1st and 99th percentiles.

\section{Baseline covariates}

Potential confounders of the association between disability and mental health were included as covariates. This included all baseline variables that were common causes of both the exposure and outcome, assessed using a causal diagram (figure 1). These were measured in 2011, as a measure of people's circumstances prior to disability acquisition. Demographic characteristics included age, sex and country of birth (Australia; other) and socioeconomic characteristics included parental occupation (high skill; medium skill; low skill or not in the labour force), education (bachelor degree and above; completion of secondary education; did not complete secondary), occupation (high skill; medium skill; low skill; not employed), income (weekly personal disposable income), wealth (tertiles of net wealth), financial hardship (prosperous/ very comfortable; reasonably comfortable; just getting along/poor/very poor), housing tenure (outright owner; mortgager; renter), housing unaffordability (defined as households in the lowest $40 \%$ of the income distribution with housing costs exceeding $30 \%$ of gross income), relationship status (yes; no), children (yes; no), social support (constructed using the average of 10 questions addressing emotional support) ${ }^{35}$ frequency of socialising with friends or relatives (rated on a 7-point Likert scale ranging from less than once every 3 months to daily), smoking status (never; ex-smoker; current), alcohol consumption (never; rarely; 1-2 days per week; $>2$ days per week), physical activity ( $>3$ times per week; $1-3$ times per week; less than once a week) and body mass index $\left(\mathrm{BMI} ; \mathrm{kg} / \mathrm{m}^{2}\right)$. Baseline mental health was also included as a covariate in the models.

\section{Statistical analysis}

Interventional effects estimate average population-level effects on an outcome of shifting the distribution of a mediator in the population to what would be expected if everyone in the population was exposed. The interventional direct effect (IDE) estimates the effect on people's mental health $(\mathrm{Y})$ of acquiring a disability (A) under a stochastic intervention $G_{0}$ which shifts the distribution of employment (M1) or income (M2) in the population. It is calculated as the difference between two counterfactuals $Y_{1 G_{0}}$ and $Y_{0 G_{0}}$, where $Y_{1 G_{0}}$ represents an individual's mental health had they had a disability $(\mathrm{a}=1)$ and the mediator of interest was set to a value randomly drawn from the conditional distribution of values of the mediator among people without disability, given the observed covariates. ${ }^{22}$

It is defined as:

$$
\mathrm{IDE}=Y_{1 G_{0}}-Y_{0 G_{0}}
$$

The interventional indirect effect (IIE) estimates the effect on the outcome of equalising the distribution of the mediator, shifting the mediator from the distribution that would be expected if everyone were unexposed to that if everyone were exposed, defined as:

$$
\mathrm{IIE}=Y_{1 G_{1}}-Y_{1 G_{0}}
$$

In the presence of two causally related mediators M1 and M2, the interventional effects approach decomposes the total causal effect (TCE) of an exposure on an outcome into four components: the IDE and three IIEsthrough M1; through M2; and through the dependence of M1 and M2.

The estimation of interventional effects relies on three assumptions about confounding for estimates to have a causal interpretation:

1. No unmeasured confounding of the exposure-outcome relationship.

2. No unmeasured confounding of the mediator-outcome relationship.

3. No unmeasured confounding of the exposure-mediator relationship.

Under these assumptions, the counterfactual definitions of the TCE, IDE and IIE can be related to empirical expressions that we can estimate from data.

The TCE was estimated by fitting a linear regression model for mental health conditional on disability acquisition and baseline covariates, generating a predicted mean mental health score for each individual had they acquired a disability and had they remained disability-free.

Assuming that employment (M1) causally precedes income (M2, figure 1), the interventional effects approach enabled estimation of the IIE through employment, the IIE through income and the IIE resulting from the dependence between employment and income. Four models were fitted to the data to estimate the IDE and IIEs: (i) a logistic regression model for employment conditional on disability acquisition and baseline covariates; (ii) a linear regression model for income conditional on disability acquisition, employment and baseline covariates, including an interaction between disability acquisition and employment (model for M2 conditioning on M1); (iii) a linear regression model for income conditional on disability acquisition and baseline covariates (model for M2 marginalising over M1); and (iv) a linear regression model for mental health conditional on disability acquisition, employment, income and baseline covariates, including an interaction between disability 
acquisition and employment. The two models for income (models 2 and 3), although likely to be incompatible, enable estimation of indirect effects through different pathways (through M1, M2 and through their dependence) because conditioning on M1 allows estimation of the joint distribution of M1 and M2, whereas marginalising over M1 allows estimation of the independent distributions of M1 and M2. All models included the same set of baseline covariates, listed above and illustrated in the causal diagram (figure 1).

The IDE and IIEs were estimated using the Monte-Carlo approach described in Vansteelandt and Daniel. ${ }^{22}$ This involved sampling values of the mediators for each individual by taking 1 million random draws from the fitted distributions of the mediators for different counterfactual exposure scenarios (using models 1, 2 and 3). These sampled values were then used in model 4 to predict expected values of the outcome under different counterfactual scenarios of the exposure and mediator, which were used to estimate the IDE and IIEs. ${ }^{22}$ For each IIE, we further calculated the proportion of the effect mediated as the ratio of the IIE to the TCE. The proportion mediated captures the importance of each pathway by measuring how much of the effect of disability acquisition on mental health is due to the effect of disability on each of the mediators. ${ }^{36}$ We note that confidence intervals for the proportion mediated have been shown to be highly variable because they combine uncertainty from both the IIE and the TCE leading to larger standard errors than the estimates of the IIE. ${ }^{36}$ We would recommend drawing conclusions about the magnitude of the indirect effect from the IIE and its CI rather than the proportion mediated. Bootstrapping with 200 replications was used to calculate 95\% CIs. Analyses were conducted using Stata/ SE V.14.2. ${ }^{37}$

\section{Missing data}

Participants were included in the study if they participated in all four waves of the study. For these waves, there were missing observations for the outcome, mediators and some baseline covariates (online supplemental table S1). The variables with the greatest amount of missing data were mental health (9\%-10\%); smoking, alcohol consumption, physical activity and financial hardship $(10 \%)$; social support and frequency of socialising (11\%); and BMI (13\%).

The distribution of baseline covariates (measured in 2011) was compared between participants with and without missing observations. Participants with missing data had poorer mental health, were younger, were more likely to be born outside of Australia and experienced greater socioeconomic compared with those with complete data (online supplemental table S2), suggesting that the data were not missing completely at random.

Multiple imputation using chained equations with 50 imputations was performed to maximise the information available and reduce bias due to missing data. The imputation models included all variables in the target analysis as well as additional auxiliary variables including mental health $(2010,2012,2013)$, self-rated health (2010-2014), socioeconomic characteristics $(2010,2012)$ except education, parent occupation and wealth which changed minimally over time, and Socio-Economic Indexes for Areas (SEIFA) score (2011). The mediation analysis was conducted on each of the 50 imputed datasets, the mean of which was calculated to give an overall estimate of the total, direct and indirect effects. SEs were derived using Rubin's Rules for combining the between-imputation and within-imputation variance, with the latter obtained by bootstrapping of each imputed dataset. ${ }^{38}$ Results were compared with the complete case analysis (secondary analysis).

\section{Sensitivity analysis}

The effect on the mental health of acquiring a psychosocial impairment is likely to be different from the effect associated with other types of impairments. Therefore as a sensitivity analysis, we repeated the analysis on a subsample of the data, removing participants who acquired psychosocial impairments, defined as nervous or emotional conditions which required treatment, or any mental illness which required help or supervision.

\section{Patient and public involvement}

We did not directly include patient and public involvement in this study.

\section{RESULTS}

\section{Sample selection}

Of the 28794 people who participated in the first 14 waves of the HILDA Survey, 14534 participated in all four waves between 2011 and 2014, of which 10450 were of working age and had complete data on disability (figure 2). Based on their reported disability status in the four waves, 5652 people were eligible for inclusion in the sample: 233 (4.1\% acquired a disability) and 5419 did not report a disability in any of the waves of analysis. Complete data for the exposure, mediators, confounders and outcome were available for 4311 participants, $76 \%$ of the sample.

\section{Descriptive analysis}

Baseline demographic and socioeconomic characteristics of the sample were described for a randomly selected imputed dataset (table 1). At baseline, prior to reporting a disability, people in the disability sample were older, had lower mental health, and were more likely to experience socioeconomic disadvantage including low education; low skilled occupation, unemployment or not in the labour force; low income; financial hardship; unaffordable housing; lower social support and frequency of socialising; more likely to be current smokers; less likely to exercise and higher BMI. They were similar to those without disability in terms of sex, country of birth, parents' occupation, wealth, housing tenure, relationship status, number of children and alcohol consumption. 


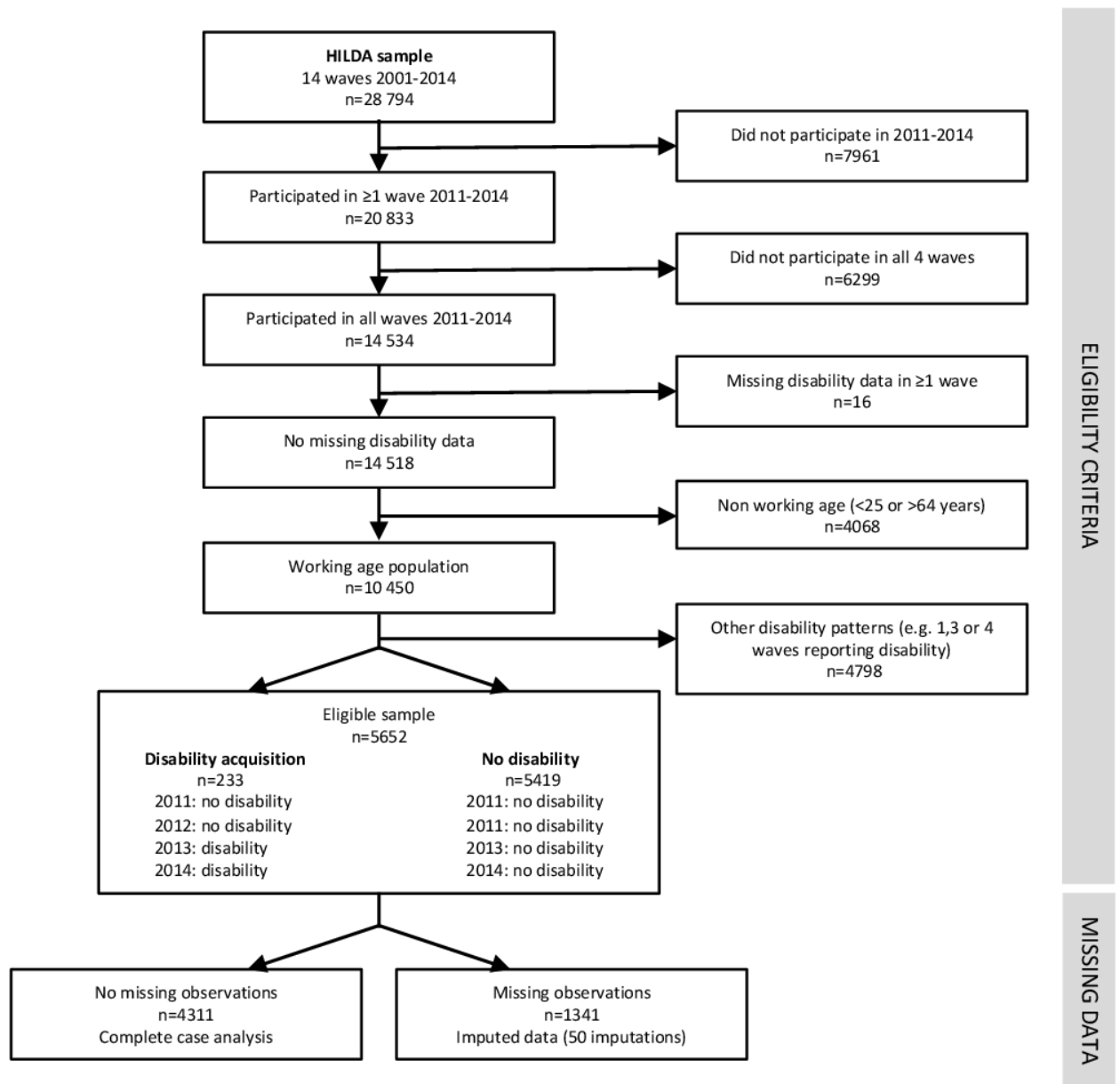

Figure 2 Flow chart illustrating sample selection and missing data. HILDA, Household, Income and Labour Dynamics in Australia.

People who acquired a disability had poorer mental health than people who did not acquire a disability (mean score of 67.9 vs 77.0 , table 2 ). They were also more likely to be unemployed or not in the labour force $(27.0 \%$ vs $14.6 \%)$ and had lower weekly disposable income ( $\$ 880.4$ vs $\$ 1057.9$ ) compared with those with no disability.

\section{Mediation analysis}

The TCE of acquiring a disability was estimated to be a 5-point decline in mental health score (estimated mean difference: $-4.8,95 \%$ CI -7.0 to -2.7 , table 3 ). The results of the four models used to estimate the IIEs and the IDE are presented in the supplementary material (online supplemental tables S3-S6). The IIE through employment was estimated as a 0.5 -point decline $(-0.5$, $95 \%$ CI -1.0 to 0.0 ), accounting for $10.6 \%$ of the total effect ( $95 \%$ CI -1.0 to 22.1 ), and there was no evidence of an indirect effect through income $(0.095 \%$ CI 0.0 to 0.1 ) nor through the dependence of employment and income $(0.0,95 \%$ CI 0.0 to 0.0$)$. The results of the complete case analysis supported the findings of the multiple imputation analysis, with a slight attenuation of the magnitude of the TCE and IDE and wider CIs (table 3).

\section{Sensitivity analysis}

The sensitivity analysis excluding participants with psychosocial impairments estimated the TCE to be slightly smaller in magnitude $(-3.8,95 \%$ CI -6.0 to -1.6 , table 4) but a similar proportion of the effect mediated by employment $(11.6 \%)$.

\section{DISCUSSION}

This study estimated that $10.6 \%$ of the effect of disability acquisition on mental health was explained by changes to people's employment but none through income. Interventional effects estimate the effect on mental health of equalising the distribution of employment for people with and without a disability. Therefore, this study estimated that disability-related mental health inequalities could be reduced by $10.6 \%$ if employment rates were the same for people with disabilities as those without.

Disability acquisition is likely to lead to a decline in people's mental health through a variety of mechanisms. A previous analysis using the same data suggested that material socio-economic factors accounted for $32 \%$ of the total effect of disability acquisition on mental health. The findings of this study suggest that employment status 
Open access

Table 1 Distribution of baseline demographic, socioeconomic and mental health characteristics for people who acquired a disability and those with no disability for a randomly selected imputed dataset

\begin{tabular}{|c|c|c|c|c|}
\hline \multirow[b]{2}{*}{ Categorical variables } & \multicolumn{2}{|c|}{ No disability $(n=5419)$} & \multicolumn{2}{|c|}{ Disability (n=233) } \\
\hline & n & $\%$ & $\mathbf{n}$ & $\%$ \\
\hline \multicolumn{5}{|l|}{ Sex } \\
\hline Men & 2607 & 48.1 & 117 & 50.2 \\
\hline Women & 2812 & 51.9 & 116 & 49.8 \\
\hline \multicolumn{5}{|l|}{ Country of birth } \\
\hline Australia & 4116 & 76.0 & 181 & 77.7 \\
\hline Other & 1303 & 24.1 & 52 & 22.3 \\
\hline \multicolumn{5}{|l|}{ Parent occupation } \\
\hline High skilled & 812 & 15.0 & 43 & 18.5 \\
\hline Medium skilled & 1817 & 33.5 & 80 & 34.3 \\
\hline Low skilled/not in the labour force & 2790 & 51.5 & 110 & 47.2 \\
\hline \multicolumn{5}{|l|}{ Education } \\
\hline Higher education & 1906 & 35.2 & 46 & 19.7 \\
\hline Secondary education & 2611 & 48.2 & 120 & 51.5 \\
\hline Less than secondary & 902 & 16.7 & 67 & 28.8 \\
\hline \multicolumn{5}{|l|}{ Employment status } \\
\hline Employed & 4698 & 86.7 & 186 & 79.8 \\
\hline Unemployed/not in the labour force & 721 & 13.3 & 47 & 20.2 \\
\hline \multicolumn{5}{|l|}{ Occupation } \\
\hline High skilled & 2119 & 39.1 & 65 & 27.9 \\
\hline Medium skilled & 1780 & 32.9 & 77 & 33.1 \\
\hline Low skilled & 799 & 14.7 & 44 & 18.9 \\
\hline Unemployed/not in the labour force & 721 & 13.3 & 47 & 20.2 \\
\hline \multicolumn{5}{|l|}{ Wealth } \\
\hline Highest tertile & 2088 & 38.5 & 84 & 36.1 \\
\hline Middle tertile & 1998 & 36.9 & 84 & 36.1 \\
\hline Lowest tertile & 1333 & 24.6 & 65 & 27.9 \\
\hline \multicolumn{5}{|l|}{ Financial hardship } \\
\hline Prosperous/very comfortable & 978 & 18.1 & 26 & 11.2 \\
\hline Reasonably comfortable & 2959 & 54.6 & 122 & 52.4 \\
\hline Just getting along/poor/very poor & 1482 & 27.4 & 85 & 36.5 \\
\hline \multicolumn{5}{|l|}{ Housing tenure } \\
\hline Outright owner & 1173 & 21.7 & 61 & 26.2 \\
\hline Mortgage & 2638 & 48.7 & 102 & 43.8 \\
\hline Renter & 1608 & 29.7 & 70 & 30.0 \\
\hline \multicolumn{5}{|l|}{ Housing affordability } \\
\hline Affordable & 4983 & 92.0 & 206 & 88.4 \\
\hline Not affordable & 436 & 8.1 & 27 & 11.6 \\
\hline \multicolumn{5}{|l|}{ Relationship status } \\
\hline In a relationship & 4280 & 79.0 & 185 & 79.4 \\
\hline Not in a relationship & 1139 & 21.0 & 48 & 20.6 \\
\hline \multicolumn{5}{|l|}{ Children } \\
\hline Yes & 1479 & 27.3 & 53 & 22.8 \\
\hline No & 3940 & 72.7 & 180 & 77.3 \\
\hline
\end{tabular}


Table 1 Continued

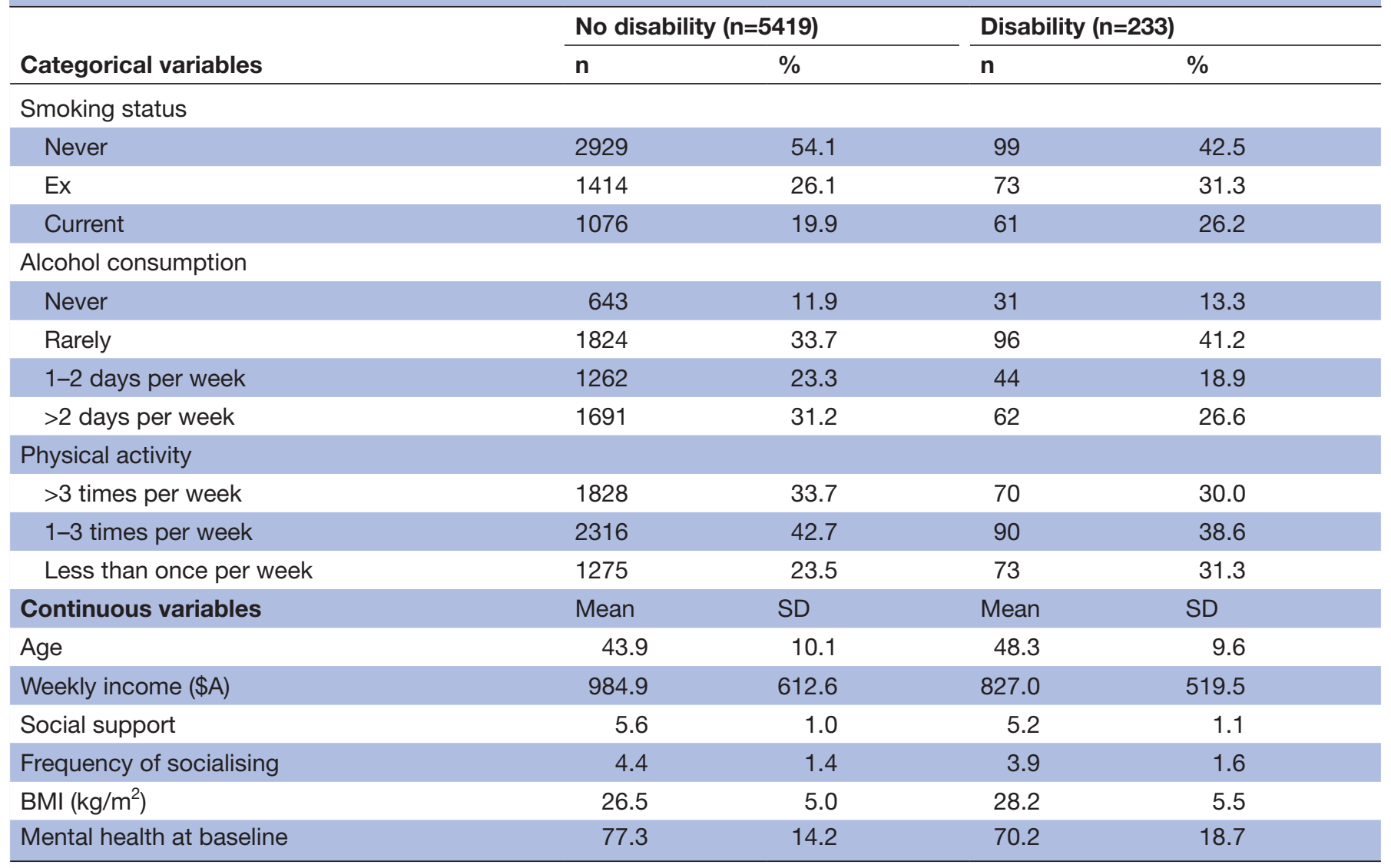

BMI, body mass index.

is a key factor in this pathway, explaining $11 \%$ of the total effect and a third of the effect explained by material socioeconomic factors. There is evidence from the literature that acquiring a disability is associated with decreasing employment rates and increased risk of belonging to low-income households, ${ }^{34}$ exposing people to higher levels of financial stress. This study suggests that changes to people's employment explained a proportion of the effect but not income, suggesting that the benefits of employment to mental health operate through pathways other than income. It may be that the positive effects of employment on mental health result from latent benefits such as social contact and social identity. ${ }^{39}$

This analysis applies a novel approach which addresses the limitations of other mediation methods. It estimates IIEs which are not defined in terms of cross-world counterfactuals, logically incompatible exposure states which can therefore neither be enforced experimentally nor tested empirically, ${ }^{40}$ and can therefore be identified under weaker assumptions than natural indirect effects. Interventional effects are particularly attractive for the analysis of multiple mediators because they allow quantification of

Table 2 Distribution of mental health (2014), employment status (2013) and income (2013) for people who acquired a disability and those with no disability for a randomly selected imputed dataset

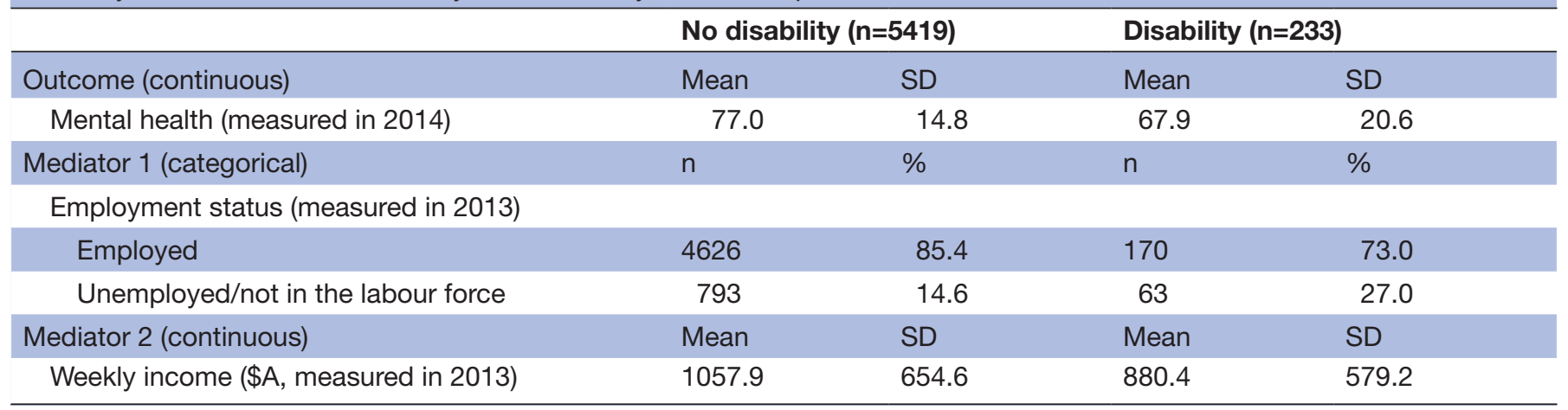


Table 3 Results of the interventional mediation analyses estimating total, direct and indirect effects of disability acquisition on mental health, with mediation through employment (M1) and income (M2)

\begin{tabular}{|c|c|c|c|}
\hline & Coeff & $95 \% \mathrm{Cl}$ & $\%$ mediated $(95 \% \mathrm{Cl})$ \\
\hline \multicolumn{4}{|l|}{ Multiple imputation } \\
\hline TCE & -4.8 & -7.0 to -2.7 & \\
\hline IIE through employment (M1) & -0.5 & -1.0 to 0.0 & $10.6(-1.0$ to 22.1$)$ \\
\hline IIE through income (M2) & 0.0 & 0.0 to 0.1 & $-0.5(-1.6$ to 0.6$)$ \\
\hline \multicolumn{4}{|l|}{ Complete case analysis } \\
\hline TCE & -4.1 & -6.4 to -1.8 & \\
\hline IDE & -3.6 & -5.2 to -0.9 & \\
\hline IIE through employment (M1) & -0.7 & -1.4 to 0.0 & $17.0(-1.4$ to 35.3$)$ \\
\hline IIE through income (M2) & 0.0 & -0.1 to 0.1 & $0.0(-3.1$ to 3.2$)$ \\
\hline
\end{tabular}

IDE, interventional direct effect; IIE, interventional indirect effect; TCE, total causal effect.

effects via distinct pathways even if the structural dependence between mediators is unknown. ${ }^{22}$ Furthermore, interventional effects quantify average population-level effects representing the effect of shifting the distribution of the mediator in the population to what would be expected if everyone in the population was exposed. This population average effect is a more realistic estimate than natural effects which estimate individual causal effects. ${ }^{23}$

There were also a number of strengths relating to the data and the analysis. The longitudinal nature of the data was used to establish a temporal sequence between the baseline variables, exposure, mediators and outcomes to better model the causal relationships. This analysis examined incident disability, comparing people who acquired a disability to those who reported no disability, to account for differences between the groups prior to disability to maximise causal inference. It also adjusted for baseline values of the mediators and baseline mental health so that the indirect effects can be interpreted as the effect of changes in the mediators as a result of disability acquisition. There were a number of limitations. Causal mediation analysis relies on strong assumptions about confounding for the estimates to have a causal interpretation. ${ }^{36}$ This analysis controlled for a large number of baseline variables, measured prior to disability acquisition, which should have accounted for differences in measured variables between the exposure groups. However, it is possible that there may have been some mediator-outcome confounding. There were only 233 people who acquired a disability in our dataset, which resulted in low power to detect effects, particularly when partitioning the effect into direct and indirect effects through additional variables. Employment was examined as a binary variable due to limitations of the methods which cannot model categorical mediators. This coarse classification of employment may have underestimated the indirect effect through employment. We examined mediators in 2013, in the same wave of data that people first reported a disability. It is possible that changes to employment and income resulting from disability acquisition may become apparent prior or subsequent to 2013 than we examined in this analysis, which may have led to an underestimate of the effect operating through employment and income. However, the biggest change in the distribution of employment status and income for individuals in the sample who acquired a disability occurred in 2013, therefore it is unlikely to have substantially biased the results. All variables were self-reported

Table 4 Results of the sensitivity analysis excluding people with psychosocial impairments

\begin{tabular}{lccc}
\hline & Coeff & $95 \% \mathbf{C l}$ & \% mediated \\
\hline Multiple imputation & & & \\
TCE & -3.8 & -6.0 to -1.6 & -5.2 to -0.9 \\
IDE & -3.0 & -0.9 to 0.1 & $11.6(-16.2$ to 39.4$)$ \\
IIE through employment (M1) & -0.4 & 0.0 to 0.1 & $-0.4(-2.9$ to 2.2$)$ \\
IIE through income (M2) & 0.0 & 0.0 to 0.0 & $0.0(-0.3$ to 0.2$)$ \\
IIE from dependence of M1 and M2 & 0.0 & & 0.0 \\
\hline
\end{tabular}

IDE, interventional direct effect; IIE, interventional indirect effect; TCE, total causal effect. 
and therefore prone to measurement error, which may have introduced bias if the degree of error was different between the exposure groups. There were missing data for $24 \%$ of participants in the sample, however, we used multiple imputation which is likely to have controlled for selection bias from missing data. Finally, the HILDA sampling frame included residents of private dwellings. People with severe disabilities, who may be more likely to live in non-private dwellings such as nursing homes or group homes, are likely to be under-represented in the sample.

The finding that disability-related mental health inequalities could be reduced by approximately $11 \%$ by equalising employment rates between people with and without disabilities has important implications. The results suggest that employment status is a mediator of the relationship between disability acquisition and mental health and that increasing employment rates for people with disability may reduce mental health inequalities. Further research is needed to understand how other aspects of employment contribute to the relationship as well as studies with larger sample sizes given the large uncertainty in the estimates of indirect effects.

\section{Author affiliations}

'Disability and Health Unit, Centre for Health Equity, Melbourne School of Population and Global Health, The University of Melbourne, Parkville, Victoria, Australia ${ }^{2}$ Biostatistics Unit, Centre for Epidemiology and Biostatistics, Melbourne School of Population and Global Health, The University of Melbourne, Parkville, Victoria, Australia

${ }^{3}$ Healthy Housing Unit, Centre for Health Policy, Melbourne School of Population and Global Health, The University of Melbourne, Parkville, Victoria, Australia

\section{Twitter Zoe Aitken @Aitken and Anne Marie Kavanagh @Kavanagh_AM}

Acknowledgements This paper uses unit record data from the Household, Income and Labour Dynamics in Australia (HILDA) Survey. The HILDA project was initiated and is funded by the Australian Government Department of Social Services (DSS) and is managed by the Melbourne Institute of Applied Economic and Social Research (Melbourne Institute). The findings and views reported in this paper, however, are those of the author and should not be attributed to either DSS or the Melbourne Institute.

Contributors ZA conceived the research question, conducted the analysis, interpreted the results and prepared the manuscript. JAS, RB and AMK made substantial contributions to the study conception, the data analysis methods, the interpretation of results and critically reviewed the manuscript. All authors read and approved the final manuscript. ZA is the guarantor of this paper and accepts full responsibility for the work

Funding This work was supported by an Australian Government Research Training Programme Scholarship and a National Health and Medical Research Council Postgraduate Scholarship (1093740) to ZA, a National Health and Medical Research Council Investigator Grant (1196068) to JAS, an Australian Research Council Future Fellowship (FT15010013) to RB and a National Health and Medical Research Council Centre of Research Excellence (1116385).

Competing interests None declared.

Patient consent for publication Not applicable.

Ethics approval This study was approved by the Melbourne School of Population and Global Health Human Ethics Advisory Group (ID 1545138.1).

Provenance and peer review Not commissioned; externally peer reviewed.

Data availability statement Data may be obtained from a third party and are not publicly available. Data are available to researchers through the National Centre for Longitudinal Data Dataverse (https://dataverse.ada.edu.au/dataverse/ ncld).
Supplemental material This content has been supplied by the author(s). It has not been vetted by BMJ Publishing Group Limited (BMJ) and may not have been peer-reviewed. Any opinions or recommendations discussed are solely those of the author(s) and are not endorsed by BMJ. BMJ disclaims all liability and responsibility arising from any reliance placed on the content. Where the content includes any translated material, BMJ does not warrant the accuracy and reliability of the translations (including but not limited to local regulations, clinical guidelines, terminology, drug names and drug dosages), and is not responsible for any error and/or omissions arising from translation and adaptation or otherwise.

Open access This is an open access article distributed in accordance with the Creative Commons Attribution Non Commercial (CC BY-NC 4.0) license, which permits others to distribute, remix, adapt, build upon this work non-commercially, and license their derivative works on different terms, provided the original work is properly cited, appropriate credit is given, any changes made indicated, and the use is non-commercial. See: http://creativecommons.org/licenses/by-nc/4.0/.

ORCID iDs

Zoe Aitken http://orcid.org/0000-0002-5413-2450

Rebecca Bentley http://orcid.org/0000-0003-3334-7353

Anne Marie Kavanagh http://orcid.org/0000-0002-1573-3464

\section{REFERENCES}

1 ABS. Disability, ageing and carers: summary of findings, 2018 (4430.0. Canberra: ABS, 2019.

2 Kavanagh AM, Krnjacki L, Kelly M. Disability and health inequalities in Australia: research summary: addressing the social and economic determinants of mental and physical health. Melbourne: VicHealth, 2012.

3 Australian Institute of Health and Welfare. Health of Australians with disability: health status and risk factors. Bulletin 83 Cat no. AUS 132. Canberra: AlHW, 2010.

4 Krahn GL, Walker DK, Correa-De-Araujo R. Persons with disabilities as an unrecognized health disparity population. Am J Public Health 2015;105 Suppl 2:S198-206.

5 Altman BM, Bernstein A. Disability and health in the United States, 2001-2005. Hyattsville, MD: National Center for Health Statistics, 2008.

6 Ormel J, Rijsdijk FV, Sullivan M, et al. Temporal and reciprocal relationship between IADL/ADL disability and depressive symptoms in late life. J Gerontol B Psychol Sci Soc Sci 2002;57:P338-47.

7 Yang Y. How does functional disability affect depressive symptoms in late life? the role of perceived social support and psychological resources. J Health Soc Behav 2006;47:355-72.

8 Lucas RE. Long-Term disability is associated with lasting changes in subjective well-being: evidence from two nationally representative longitudinal studies. J Pers Soc Psychol 2007;92:717-30.

9 Aitken Z, Krnjacki L, Kavanagh AM, et al. Does social support modify the effect of disability acquisition on mental health? A longitudinal study of Australian adults. Soc Psychiatry Psychiatr Epidemiol 2017;52:1247-55.

10 Aitken Z, Simpson JA, Bentley R, et al. Disability acquisition and mental health: effect modification by demographic and socioeconomic characteristics using data from an Australian longitudinal study. BMJ Open 2017;7:e016953.

11 Kavanagh AM, Aitken Z, Baker E, et al. Housing tenure and affordability and mental health following disability acquisition in adulthood. Soc Sci Med 2016;151:225-32.

12 Kavanagh AM, Aitken Z, Krnjacki L, et al. Mental Health Following Acquisition of Disability in Adulthood--The Impact of Wealth. PLoS One 2015;10:e0139708.

13 Mandemakers JJ, Monden CWS. Does education buffer the impact of disability on psychological distress? Soc Sci Med 2010;71:288-97.

14 Bruce ML. Depression and disability in late life: directions for future research. Am J Geriatr Psychiatry 2001;9:102-12.

15 Smith DM, Langa KM, Kabeto MU, et al. Health, wealth, and happiness: financial resources buffer subjective well-being after the onset of a disability. Psychol Sci 2005;16:663-6.

16 Kariuki M, Honey A, Emerson E, et al. Mental health trajectories of young people after disability onset. Disabil Health J 2011;4:91-101.

17 Emerson E, Kariuki M, Honey A, et al. Becoming disabled: the association between disability onset in younger adults and subsequent changes in productive engagement, social support, financial hardship and subjective wellbeing. Disabil Health $J$ 2014;7:448-56.

18 Yang Y, George LK. Functional disability, disability transitions, and depressive symptoms in late life. J Aging Health 2005;17:263-92. 
19 Miller LS. Physical Illness and Depression in Older Adults: A Summary with Implications for Future Directions in Research and Treatment. In: Williamson GM, Shaffer DR, Parmelee PA, eds. Physical illness and depression in older adults: a Handbook of theory, research, and practice. New York: Plenum, 2000.

20 Aitken Z, Simpson JA, Gurrin L, et al. Do material, psychosocial and behavioural factors mediate the relationship between disability acquisition and mental health? a sequential causal mediation analysis. Int J Epidemiol 2018;47:829-40.

21 Hafeman DM, Schwartz S. Opening the black box: a motivation for the assessment of mediation. Int J Epidemiol 2009;38:838-45.

22 Vansteelandt S, Daniel RM. Interventional effects for mediation analysis with multiple mediators. Epidemiology 2017;28:258-65.

23 Moreno-Betancur M, Carlin JB. Understanding interventional effects: a more natural approach to mediation analysis? Epidemiology 2018;29:614-7.

24 Ware JE, Kosinski M, Gandek B. SF-36 health survey: manual \& interpretation guide. Lincoln, Rl: QualityMetric, 2000.

25 Pirkis J, Burgess P, Kirk P. Review of standardised measures used in the National outcomes and casemix collection (NOCC. Sydney: NSW Institute of Psychiatry, 2005.

26 McCabe CJ, Thomas KJ, Brazier JE, et al. Measuring the mental health status of a population: a comparison of the GHQ-12 and the SF-36 (MHI-5). Br J Psychiatry 1996;169:517-21.

27 Strand $\mathrm{BH}$, Dalgard OS, Tambs K, et al. Measuring the mental health status of the Norwegian population: a comparison of the instruments SCL-25, SCL-10, SCL-5 and MHI-5 (SF-36). Nord J Psychiatry 2003;57:113-8.

28 Weinstein MC, Berwick DM, Goldman PA, et al. A comparison of three psychiatric screening tests using receiver operating characteristic (ROC) analysis. Med Care 1989;27:593-607.

29 Yamazaki S, Fukuhara S, Green J. Usefulness of five-item and threeitem mental health inventories to screen for depressive symptoms in the general population of Japan. Health Qual Life Outcomes 2005;3:48.

30 Knox SA, King MT. Validation and calibration of the SF-36 health transition question against an external criterion of clinical change in health status. Qual Life Res 2009;18:637-45.

31 McHorney CA, Ware JE, Raczek AE. The mos 36-Item short-form health survey (SF-36): II. psychometric and clinical tests of validity in measuring physical and mental health constructs. Med Care 1993;31:247-63.

32 Burchardt T. Being and becoming: Social exclusion and the onset of disability. London: The London School of Economics, 2003.

33 Jenkins SP, Rigg JA. Disability and disadvantage: selection, onset, and duration effects. J Soc Policy 2004;33:479-501.

34 Polidano C, Vu H. Differential labour market impacts from disability onset. Health Econ 2015;24:302-17.

35 Milner A, Krnjacki L, Butterworth P, et al. The role of social support in protecting mental health when employed and unemployed: a longitudinal fixed-effects analysis using 12 annual waves of the HILDA cohort. Soc Sci Med 2016;153:20-6.

36 VanderWeele TJ. Explanation in Causal Inference: Methods for Mediation and Interaction. New York: Oxford University Press, 2015.

37 StataCorp. Stata statistical software: release 14. College Station, TX: StataCorp LP, 2015.

38 Rubin D. Multiple imputation for nonresponse in surveys. New York: Wiley, 1987.

39 Jahoda M. Employment and unemployment: A social-psychological analysis. Cambridge: Cambridge University Press, 1982.

40 Pearl J. Direct and indirect effects. Proceedings of the Seventeenth Conference on Uncertainty in Artificial Intelligence, 2001: 411-20.

41 Robins JM, Richardson TS. Alternative graphical causal models and the identification of direct effects. In: Shrout P, ed. Causality and psychopathology: finding the determinants of disorders and their cures. Oxford: Oxford University Press, 2010: 103-58. 